\title{
Radiation Resistance of Tar Fractions of Bituminous Oil
}

\author{
Nigar Guliyeva*, Islam Mustafayev, Saida Mamedova and Samira Aliyeva \\ Institute of Radiation Problems of the Azerbaijan National Academy of Sciences, Baku AZ1143, Azerbaijan
}

\begin{abstract}
The results of studies of radiation chemical transformations of Balakhani bituminous oil of Azerbaijan are adduced. The IR (infrared) spectra of initial and irradiated samples of tar fractions of bituminous oil are compared. The kinetics of radiation chemical yields of gas products at the irradiation of bituminous oil and its tar fraction have been investigated. Irradiation is conducted in the gamma-ray source of isotope ${ }^{60} \mathrm{Co}$ at the dose rate of $\mathrm{P}=0.27 \mathrm{~Gy} / \mathrm{s}$ and absorbed doses $\mathrm{D}=5-163 \mathrm{kGy}$. It is found that in comparison with oil faction, tar fraction of bituminous oil has a high resistance to radiation.
\end{abstract}

Key words: Bituminous oil, tar faction, $\gamma$-radiolysis, radiation resistance, IR spectra, chromatography, gas products.

\section{Introduction}

One of the most important quality characteristics of materials intended for use in industrial production is their radiation resistance, the ability to withstand the effects of radiation and preserve the original properties [1]. From this point of view the study of radiation resistance of bituminous oils and petroleum fractions has a practical interest. Thus development of non-traditional technology to production of radiation-resistant petroleum compounds requires investigation of the mechanism of occurring destruction and polycondensation processes during their radiolysis of petroleum and his fractions.

This paper presents the results of the study of radiation resistance of tar fraction of Balakhani bituminous oil of Azerbaijan. The content of tar-asphaltene compounds of oil is determined according to QOST 11858-66.

\section{Experimental Sections}

The main indicators of the radiation resistance were studied kinetics of gases formation in the $\gamma$-radiolysis of factions of heavy bituminous oil and change of concentrations of the various categories of oil compounds (total petroleum hydrocarbons, polycyclic

\footnotetext{
*Corresponding author: Nigar Guliyeva, Ph.D., research filed: radiation technology of organic fuels. E-mail: nigarguliyeva64@mail.ru.
}

aromatic compounds with the number of rings from 2 to 6 category of polycyclic aromatic components, defined by Environmental Protection Agency of US as 16 EPA, NPD (naphthalene-phenantrene-dibenzothiophene) and also change of structural group composition of liquid products of the radiolysis.

To study the material composition of the oil, namely oil, tar, and asphaltenes fractions, and the filtrate obtained after the precipitation of oil samples with n-heptane the asphaltene content was determined. The tar dissolved in the filtrate were adsorbed on silicagel and then stripped of alcohol-benzene mixture and quantified after distillation of the solvent is determined [2].

The source of ionizing radiation used isotope gamma radiation source ${ }^{60} \mathrm{Co}$ "MRX- $\gamma-30$ ". The dose rate of the radiation source is $0.27 \mathrm{~Gy} / \mathrm{s}$, the absorbed doses of 5-163 kGy. Gas products were analyzed by gas chromatography; liquid products were identified by chromatography-mass spectrometry for the apparatus GC/MS Trace DSQ (Thermo Electron, Finngan USA 2005), to determine the structural group composition of the components used by FTIR (Fourier transform infrared spectroscopy).

\section{Results and Discussion}

Radiation resistance of organic materials is made to 
determine the magnitude of the radiation chemical yield of radiolysis products.

One of the essential indicators of radiation resistance of petroleum fractions is the radiation chemical yields of gases in the processes of radiolysis [3]. They were identified and the rate of formation of radiation chemical yields of gases formed as a result of $\gamma$-radiolysis oil fractions: $\mathrm{H}_{2}, \mathrm{CO}, \mathrm{CH}_{4}, \mathrm{C}_{2} \mathrm{H}_{4}, \mathrm{C}_{2} \mathrm{H}_{6}$, $\Sigma \mathrm{C}_{3}-\mathrm{C}_{6}$ ( $\Sigma$ means the total number of gas yield with the same amount of carbon-simply, propane-propylene). As an example, Fig. 1 shows the kinetics of hydrogen formation in the radiolysis of bituminous oil fractions.

From the kinetics of formation of gas calculated mean values (at different times of exposure) radiation chemical yields of gases (Table 1).

As seen from the table, the most resistant to radiation during formation of gases have asphaltenes, tar and oils further. Radiation resistance of petroleum fractions according to the gas formation in the studied conditions is in the following ratio: asphalt: tar: oil $=13: 5: 1$.

A significant difference in the radiation resistance of these fractions is due to that their composition and structure are very different. So the content of the tar and asphaltene are more conjugated double bonds and aromatic rings and heterocyclic condensed polycyclic component, which increases its resistance to radiation.

In order to determine the mechanism of physical and chemical processes occurring in the radiolysis of various factions of bituminous oil, the radiation chemical transformations of liquid fractions were studied.

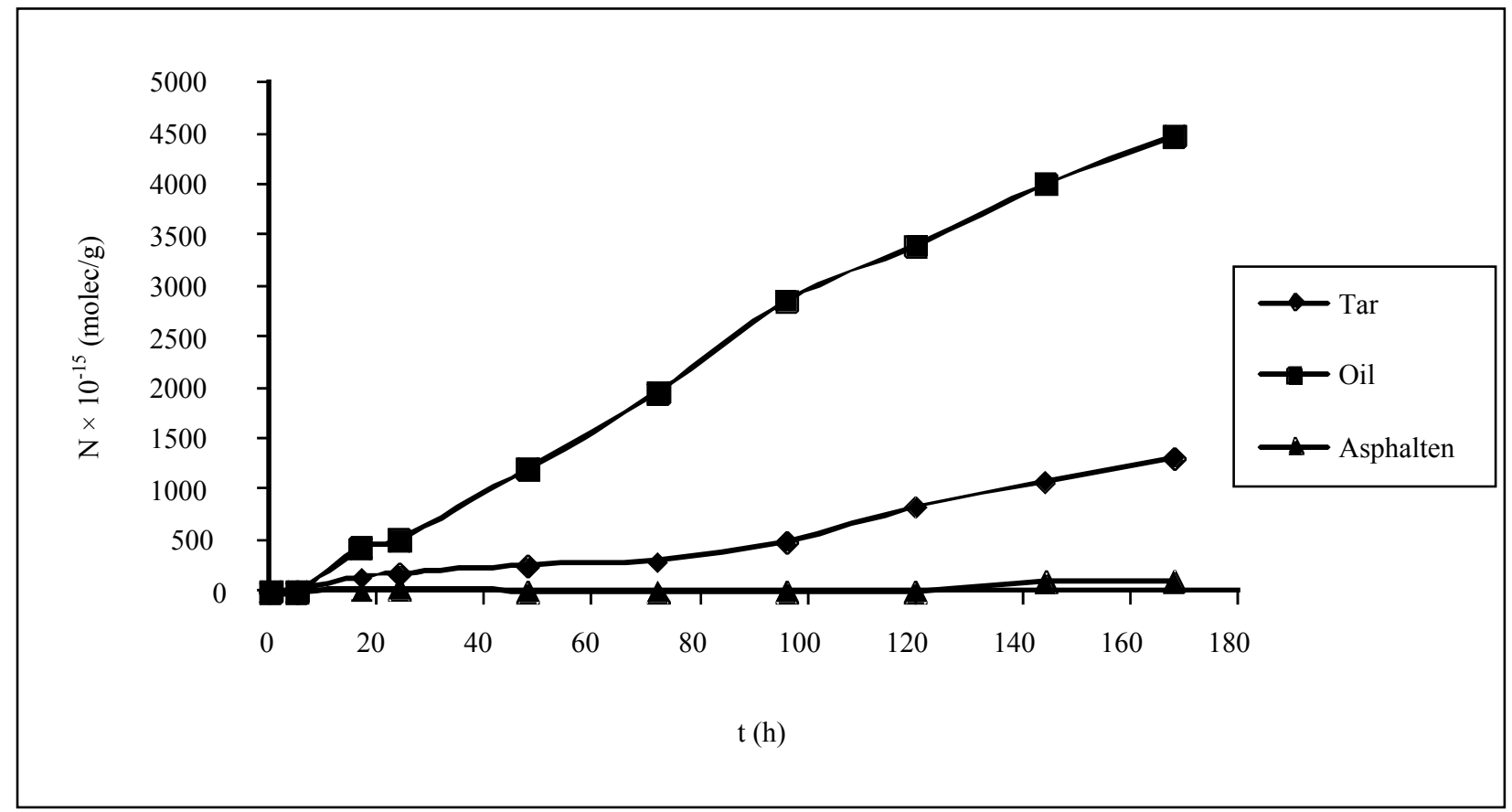

Fig. 1 The kinetics of hydrogen formation in the radiolysis of oil, tar and asphaltene fractions of bituminous oil of Balakhani oil-field.

Table 1 The average values of radiation chemical yields of gases.

\begin{tabular}{llllllllll}
\hline \multirow{2}{*}{ Fractions } & \multicolumn{10}{c}{$\mathrm{G}, \mathrm{molec} / 100 \mathrm{eV}$} \\
\cline { 2 - 10 } & $\mathrm{H}_{2}$ & $\mathrm{CH}_{4}$ & $\mathrm{C}_{2} \mathrm{H}_{6}$ & $\mathrm{C}_{2} \mathrm{H}_{4}$ & $\Sigma \mathrm{C}_{3}$ & $\Sigma \mathrm{C}_{4}$ & $\Sigma \mathrm{C}_{5}$ & $\Sigma \mathrm{C}_{6}$ & $\Sigma$ \\
\hline Oil & 0.452 & 0.014 & 0.011 & 0.0028 & 0.0068 & 0.0027 & 0.00072 & 0 & 0.49 \\
Tar & 0.092 & 0.005 & 0.0036 & 0.00082 & 0.0018 & 0.0015 & 0.0003 & 0.0056 & 0.11 \\
Asphaltene & 0.0195 & 0.0031 & 0.0048 & 0.0011 & 0.0043 & 0.003 & 0.0006 & 0 & 0.036 \\
\hline
\end{tabular}

$\Sigma$ : The amount of radiation chemical yields of gas oil fractions in the radiolysis. 
Molecules of polycyclic components studied tars constructed from blocks in which include the identical structural types, homo- and heteroaromatic rings, polinaftenoaromatic and polycyclic systems.

The results of the mass spectrometric investigation of the aromatic part of Balakhani bituminous oil showed that in the all heavy fractions are present various PAHs, naphthalene, acenaphthene, phenanthrene, pyrene, chrysene and fluorene [4]. The kinetics of changes of concentrations of the compounds - total hydrocarbons, polycyclic aromatic (with the number of rings from 2 to 6), $16 \mathrm{EPA}$, NPD and individual polynuclear hydrocarbons in the fractions by irradiation was experimentally established.

Fig. 2 shows the kinetics of changes in the concentration of polycyclic aromatic compounds (number of rings 2-6) in the radiolysis of tar fraction.

In contrast to oil fractions, which even in irradiating the samples to $163 \mathrm{kGy}$ changes were not observed, radiolysis of petroleum tar leads to significant changes in the concentration of polycyclic aromatic compounds. This can be explained, apparently splitting and dehydration cyclic structures tar by means of radiation [5].

For example, in the range of absorbed dose of gamma irradiation of tar fraction to $163 \mathrm{kGy}$ reduced concentrations observed following compounds: common hydrocarbons-26\%, $16 \mathrm{PAH}-78 \%, 2-6$ aromatic rings-70\%, the naphthalene-phenanthrene-dibenzothiophene-71\%.

The difference in change in concentration of these oil compounds is explained by characteristics of distribution of radiation energy between the polycyclic aromatic compounds. Aromatic hydrocarbons are very stable to the effects of radiation. This is due to the fact that the excitation energy originally arising in the primary act of ionization and excitation in any particular fragment of the aromatic molecules due to the presence $\pi$-bonds are delocalized in the aromatic ring. As a result, none of the vibration degrees of freedom, does not get enough energy to break the chemical bond, and the gap of the ring becomes unlikely.

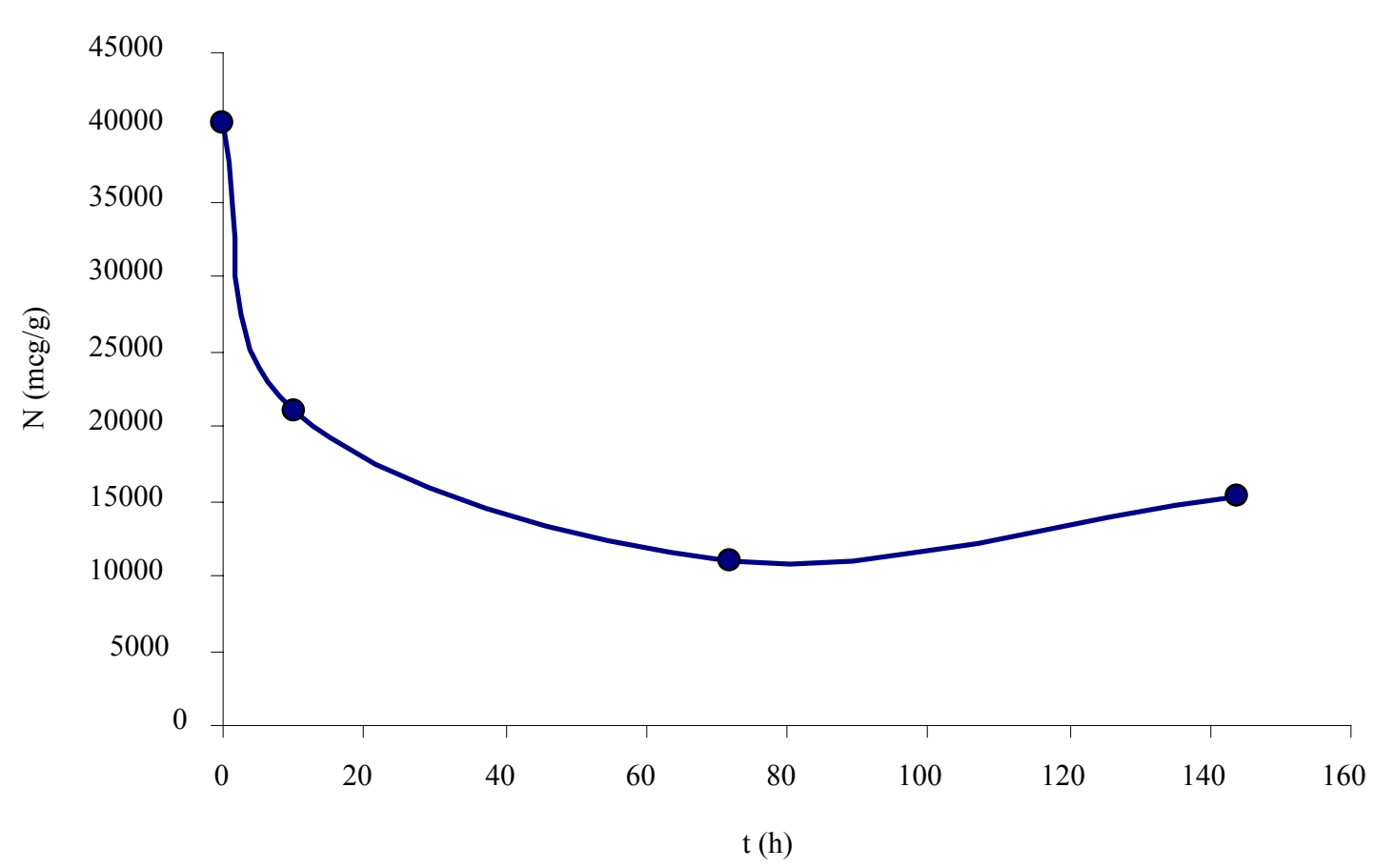

Fig. 2 Kinetics of changes in the concentration of 2-6 ring polycyclic aromatic hydrocarbons in the radiolysis of tar fraction of bituminous oil. 
In order to explain the mechanism of these processes the kinetics of changes of concentrations of the individual components with fused aromatic rings in the oil at the radiation impact has been studied.

Fig. 3 shows the kinetics of changes the concentration of polynuclear compounds, fluorine, phenanthrene, and chrysene in the tar composition.

The observed significant changes in the concentrations of these compounds in the radiolysis of the tar fraction are as follows: fluorine- $50 \%$, chrysene- $90 \%$, fenantren $-69 \%$.

The effects of gamma irradiation on the structural-group composition of tar fraction have been studied by the IR spectroscopy [6]. Fig. 4 shows the IR spectra of initial and irradiated samples of tar fraction.

The high content of aromatic rings in the tar fraction confirmed appreciable intensity band 1,600 $\mathrm{cm}^{-1}$. Analysis of IR spectra showed that, under the influence of gamma radiation observed changes in the intensity of the absorption bands at wavelengths 2,852-2,964 $\mathrm{cm}^{-1}$, responsible for the valence fluctuations groups $=\mathrm{CH}_{2}$ and $-\mathrm{CH}_{3}$ corresponding fused aromatic rings, and these strips deformation vibrations groups at $1,376-1,457 \mathrm{~cm}^{-1}$. The reduction of intensities of the absorption bands of deformation vibrations of $-\mathrm{CH}_{3}$ at wavelength $1,457 \mathrm{~cm}^{-1}$, and deformation vibrations of $-\mathrm{CH}$ monoaromatic rings and substituted polynuclear structures at a wavelength $811 \mathrm{~cm}^{-1}$ after irradiation happens due to dissipation of radiation energy in the functional groups.

Furthermore, in the tar fraction $1,707 \mathrm{~cm}^{-1}$, the band intensity responsible for stretching vibrations $\mathrm{C}=\mathrm{C}$ bond of the benzene (aromatic) ring, decreases under the influence of irradiation, due to the increasing degree of condensation of aromatic rings.

Thus, the absorption spectra of the tar are obvious that their containing saturated hydrogen carbon atoms, aromatic ring, and carbonyl group. The specific properties of the tar, compared with oil fractions, the content of the multiblock molecules is increased with a large number of aromatic rings [7]. High intensity of the band $1,380 \mathrm{~cm}^{-1}$ is corresponding to the methyl groups, indicating a relatively high molecular branching.

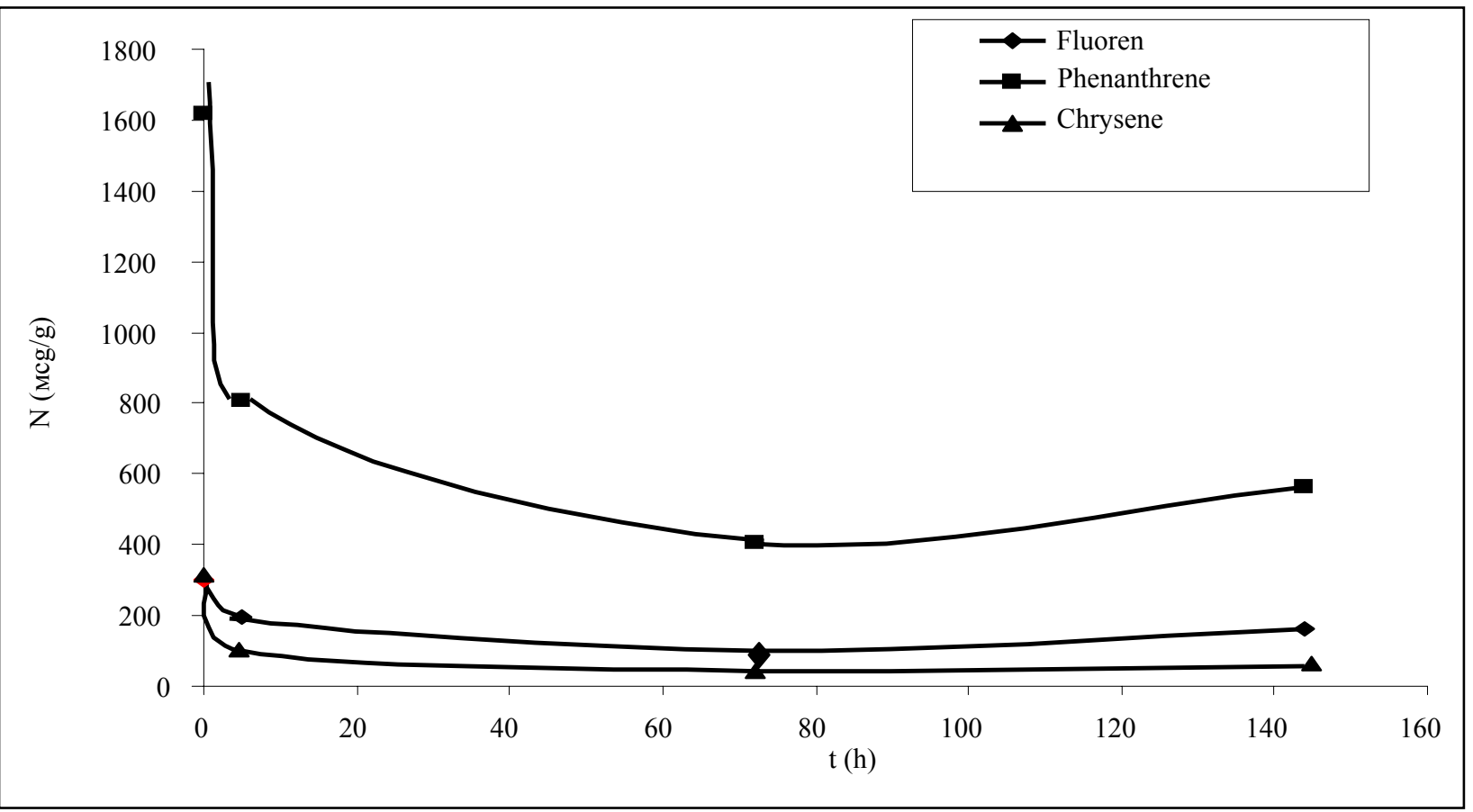

Fig. 3 Kinetics of changes of the concentration of fluorene, phenanthrene, and chrysene in the radiolysis of tar fraction of Balakhani bituminous oil. 


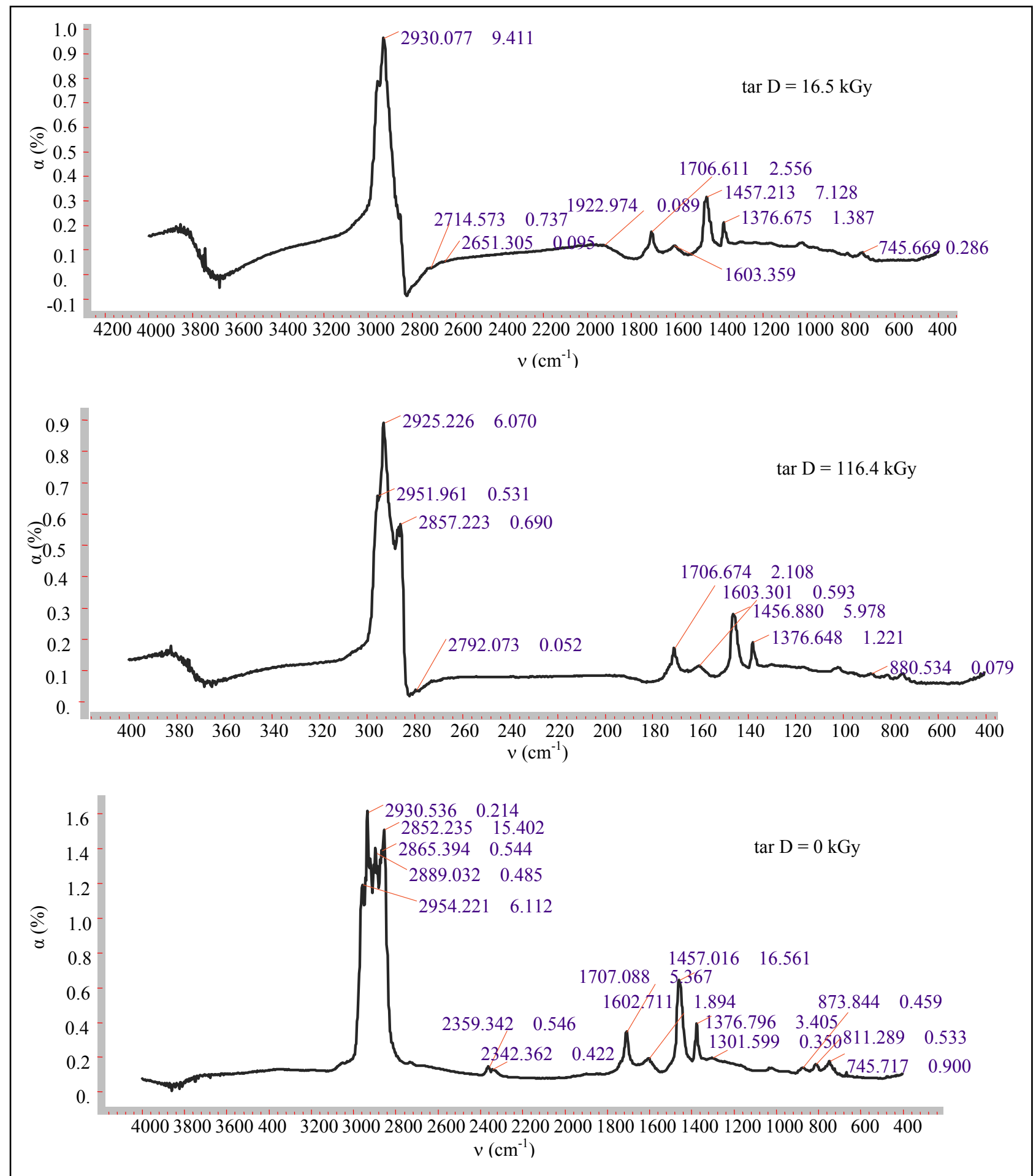

Fig. 4 IR spectras of the initial and irradiated samples of tar fraction of bituminous oil.

The hydrocarbon tar is in the form of aromatic rings with more paraffinic side chains. They are flat fused systems containing 5-6 aromatic rings, naphthenic and heterocyclic structure connected means aliphatic structures. In aromatic compounds in the transition from mono to poly-cyclic aliphatic chains are shortened. The tar is macromolecular organic compounds and heterocyclic structure has a high degree of condensation interconnected aliphatic chains. 


\section{Conclusions}

In recent years there has been an increase of heavy oil production, natural bitumen and growing interest in their high molecular weight component tar and asphaltenes because of their potential qualities as a raw material product. Face the task of control is to optimize technological processes of preparation, transportation and refining.

The effect of gamma radiation on the fractions is heavy oil bitumen, oils, tars and asphaltenes.

The experimental data show that the radiation resistance of petroleum fractions is associated with their structural group composition, and increased radiation resistance observed in tar fractions of oil, as a complex system of hydrocarbons of various structures, requires a detailed study of radiation induced transformations of petroleum fractions, the establishment of the mechanism occurring destructive and polycondensation processes at its radiolysis. This mechanism explains the radiation chemical transformations of these aromatic polyconjugated systems. The high radiation resistance feature of all cyclic compounds with unsaturated conjugated bonds. Irradiation molecules containing a benzene ring, the unpaired electron is located on the ring. In such systems with condensed aromatic rings distribution of absorbed radiation energy is a result of delocalization of $\pi$-electrons, which leads to the dissociation process and thereby expression of the radiation resistance [8].

It is found that the gamma radiolysis of petroleum fractions bituminous oil at values of absorbed radiation dose to the $\mathrm{D}=160 \mathrm{kGy}$ only in fractions of tar is observed a decrease in the concentrations of 2-6 ring polycyclic aromatic compounds with radiation chemical yields $10^{-2}-10^{-3}$ molec/100 eV because of an increase in polycondensation processes, increases the radiation resistance of the oil fractions. The absorbed dose range studied the compounds of hydrocarbon oil fractions do not undergo changes.

A significant difference in radiation resistance due to the presence of oil fractions in the composition of the fractions of tar compounds with condensed polycyclic structures. Greater stability to radiation aromatics condensed structure compared with hydrocarbons of different structure is determined by the arrangement of the alkyl chains on the aromatic ring. More stable to the action of radiation is composed of polynuclear aromatic hydrocarbon fraction of bituminous oil that can serve as a basis to recommend this class of compounds for use as a raw material in the production of building materials, as well as hydraulic fluids and lubricants even working under the action of radiation (nuclear reactors, radionuclide installation, electron accelerators, etc.).

\section{References}

[1] Milinchuk, V. K. 1986. Radiation Resistance of Organic Materials. Moscow: Energoatomizdat, pp. 272.

[2] Samedova, F. I. 2011. Azerbaijani Oil. Baku: Elm., pp. 412.

[3] Pikaev, A. K. 1986. Modern Radiation Chemistry. Radioliz of Gases and Liquids. Moscow: Nauka.

[4] Mustafayev, I. I., Aliyeva, S. F., Guliyeva, N. G., and Rzayev, R. S. 2014. "Radiation Stability of Bituminous Oils and Its Tar Fraction." Journal of Radiation Researches 1 (1): 13.

[5] Guliyeva, N., Aliyeva, S. F., Mamedov, A. P., and Yuzbashev, E. R. 2014. "The Azerbaijani Oil Industry." 07-08: 64.

[6] Nikanisi, C. 1985. Infrared Spectra and Structure of Organic Compounds. Moscow: Mir.

[7] Dmitriev, D. E. 2010. "Petrochemical". 40 (2): 118.

[8] Swallow, A. 1976. Radiation Chemistry. Moscow: Atomizdat, pp. 278. 